\title{
Some extensions and refinements of a theorem of Sylvester
}

\author{
by \\ N. Saradha (Mumbai), T. N. Shorey (Mumbai) and \\ R. TIJDEMAN (Leiden)
}

1. Introduction. Let $d>1, k \geq 3, n \geq 1$ be integers with $\operatorname{gcd}(n, d)$ $=1$. We set

$$
\Delta_{0}=\Delta_{0}(n, d, k)=n(n+d) \ldots(n+(k-1) d) .
$$

For an integer $\nu>1$, we write $\omega(\nu), P(\nu)$ and $Q(\nu)$ for the number of distinct prime divisors of $\nu$, the greatest prime factor of $\nu$ and the greatest square-free factor of $\nu$, respectively. Further we put $\omega(1)=0$ and $P(1)=Q(1)=1$.

Sylvester [11] proved that

$$
P\left(\Delta_{0}\right)>k \quad \text { if } n \geq d+k
$$

and Langevin [5] improved it to

$$
P\left(\Delta_{0}\right)>k \text { if } n>k .
$$

In fact, Sylvester and Langevin proved the above estimates also for a product of $k$ consecutive positive integers. Shorey and Tijdeman [10] showed that

$$
P\left(\Delta_{0}\right)>k \text { unless }(n, d, k)=(2,7,3) .
$$

In a recent result, Saradha and Shorey [8] showed that for $k \geq 4, \Delta_{0}$ is divisible by at least 2 distinct primes exceeding $k$ except when $(n, d, k)$ $\in\{(1,5,4),(2,7,4),(3,5,4),(1,2,5),(2,7,5),(4,7,5),(4,23,5)\}$. As to the number of prime factors of $\Delta_{0}$, Shorey and Tijdeman [9] proved that

$$
\omega\left(\Delta_{0}\right) \geq \pi(k) .
$$

A conjecture of Schinzel, known as Hypothesis $\mathrm{H}$, implies that there are infinitely many $d$ for which both $1+d$ and $1+2 d$ are primes. Thus (2) is likely to be best possible when $k=3$. Moree [6] sharpened (2) to

$$
\omega\left(\Delta_{0}\right)>\pi(k) \quad \text { if } k \geq 4 \text { and }(n, d, k) \neq(1,2,5) .
$$

2000 Mathematics Subject Classification: Primary 11B25. 
We observe that (3) implies (1) for $k \geq 4$. If $k=4$ or 5 , then as above, Hypothesis $\mathrm{H}$ implies that $\omega\left(\Delta_{0}\right)=\pi(k)+1$ for infinitely many $d$. If $k \geq 6$, we give a sharpening of (3). For this, we introduce the following notation. For any set of distinct positive integers $\left\{a_{1}, \ldots, a_{r}\right\}$ and an integer $i \geq 1$ we denote by $\left\{a_{1}, \ldots, a_{r}\right\}_{i}$ the set of distinct integers obtained by taking products of $i$ integers from $\left\{a_{1}, \ldots, a_{r}\right\}$. Let $V_{i}$ be the set of quadruples $(n, d, k, \delta)$ where $n, d, k$ equals the values in Table 1 and $\delta$ takes values from the sets \{\}$_{i}$ mentioned therein.

Table 1

\begin{tabular}{cccc}
\hline$n$ & $d$ & $k$ & $\delta=\delta_{i}$ \\
\hline 1 & 2 & 6 & $\{5,7,11\}_{i}$ \\
1 & 3 & 6 & $\{7,10,13\}_{i}$ \\
1 & 2 & 7 & $\{5,7,11,13\}_{i}$ \\
1 & 3 & 7 & $\{7,10,13,19\}_{i}$ \\
1 & 4 & 7 & $\{13,17,21\}_{i} \cup\{9,21\}_{i}$ \\
2 & 3 & 7 & $\{11,14,17\}_{i}$ \\
2 & 5 & 7 & $\{7,17,22\}_{i}$ \\
3 & 2 & 7 & $\{7,11,13\}_{i}$ \\
1 & 2 & 8 & $\{7,11,13\}_{i}$ \\
1 & 2 & 11 & $\{11,13,17,19\}_{1+i}$ \\
1 & 3 & 11 & $\{13,19,22,31\}_{1+i}$ \\
1 & 2 & 13 & $\{11,13,17,19,23\}_{1+i}$ \\
3 & 2 & 13 & $\{11,13,17,19,23\}_{1+i}$ \\
1 & 2 & 14 & $\{11,13,17,19,23\}_{1+i}$ \\
\hline
\end{tabular}

In this paper, we show

Theorem 1. Let $k \geq 6$. Then

$$
\omega\left(\Delta_{0}\right)>\frac{6}{5} \pi(k)+1
$$

except when

$$
\begin{aligned}
(n, d, k) \in\{ & (1,2,6),(1,3,6),(1,2,7),(1,3,7),(1,4,7), \\
& (2,3,7),(2,5,7),(3,2,7),(1,2,8),(1,2,11), \\
& (1,3,11),(1,2,13),(3,2,13),(1,2,14)\} .
\end{aligned}
$$

We see that Theorem 1 includes (1)-(3) with $k \geq 6$ and the result of Saradha and Shorey stated above. In Theorem 1 and the subsequent results of this paper, we observe that the statements are not valid for the exceptions mentioned therein. As a consequence of Theorem 1, we derive

Corollary 1. Let $k \geq 3$ and

$$
(n, d, k) \notin\{(1,3,3),(2,3,3),(2,7,3),(1,5,4),(3,5,4)\} .
$$


Then

$$
Q\left(\Delta_{0}\right) \geq\left(\prod_{\substack{p<k \\ \operatorname{gcd}(p, d)=1}} p\right) k^{2+\left[\frac{1}{5} \pi(k)\right]} .
$$

We observe that the second factor on the right hand side of the above inequality is $>e^{k / 5}$. Corollary 1 is related to a generalised version of a problem of Erdös and Woods (see [2]). The inequality (4) of Theorem 1 is a consequence of the following result with $t=k$. To state this result, we need some notation. Let $t \geq 2$ and $d_{1}, \ldots, d_{t}$ be distinct integers in the interval $[0, k)$. We define

$$
\Delta=\left(n, d, k, d_{1}, \ldots, d_{t}\right)=\left(n+d_{1} d\right) \ldots\left(n+d_{t} d\right)
$$

and

$$
\delta=\Delta_{0} / \Delta
$$

We observe that $\Delta=\Delta_{0}$ if $t=k$. We define $\pi_{d}(k)$ to be the number of primes $\leq k$ and coprime to $d$.

Theorem 2. Let $k \geq 6$ and $t \geq k-\frac{6}{5} \pi(k)+\pi_{d}(k)-1$. If there exist integers $d_{1}<\ldots<d_{t}$ in the interval $[0, k)$ such that

$$
\omega(\Delta) \leq \pi_{d}(k)
$$

then

$$
(n, d, k, \delta) \in V_{2} \text {. }
$$

Further, from Theorem 2 we get the following result.

Corollary 2. Let $k \geq 6, t \geq k-\frac{1}{5} \pi(k)-1$ and $\omega(\Delta) \leq \pi(k)$. Then

$$
(n, d, k, \delta) \in \begin{cases}V_{1} & \text { if }(n, d, k) \neq(1,4,7), \\ \left(1,4,7,\{13,17,21\}_{1}\right) & \text { otherwise. }\end{cases}
$$

Moree [6] showed that all solutions $(n, d, k)$ of $\omega\left(\Delta_{0}(n, d, k)\right) \leq$ $\pi(2 k-1)-2$ can be explicitly determined, and conjectured that $(n, d, k)=$ $(1,3,10)$ is the only solution. Note that $\omega\left(\Delta_{0}(1,2, k)\right)=\pi(2 k-1)-1$. Thus there exist distinct integers $d_{1}, \ldots, d_{t}$ in $[0, k)$ with $t \geq k-\pi(2 k-1)+\pi(k)+1$ and $\omega(\Delta)=\omega\left(\Delta\left(1,2, d_{1}, \ldots, d_{t}\right)\right) \leq \pi(k)$. Therefore we observe that the constant $\frac{1}{5}$ in Corollary 2 cannot be replaced by any number $\chi>1$ when $d=2$. For $d>2$ and $k$ exceeding a sufficiently large effectively computable absolute constant we show that the constant $\frac{1}{5}$ in Corollary 2 cannot be replaced by $\log (2 d)+5.2 \log \log (2 d)+5.02$. More precisely, we prove

THEOREM 3. There exist effectively computable absolute constants $C_{1}$ and $C_{2}$ such that for every triple $(n, d, k)$ satisfying

$$
n / k \leq d \leq \log k, \quad k \geq C_{1},
$$


we can find distinct integers $d_{1}, \ldots, d_{t}$ in $[0, k)$ satisfying

$$
P\left(\left(n+d_{1} d\right) \ldots\left(n+d_{t} d\right)\right) \leq k
$$

and

$$
t \geq k-\frac{k}{\log k} \bar{d}-C_{2} \frac{k \log \log k}{(\log k)^{2}} \bar{d}
$$

with

$$
\bar{d}=\log (2 d)+5.2 \log \log (2 d)+5.02 .
$$

We observe that there are infinitely many triples $(n, d, k)$ satisfying (6). A version similar to Theorem 3 was proved by Balasubramanian and Shorey $[1$, Theorem 2]. The proof of Theorem 2 is elementary whereas the proof of Theorem 3 depends on the Prime Number Theorem for arithmetic progressions with error term. Throughout the paper we shall follow the notation introduced in this section. The computations are carried out using MATHEMATICA.

2. Lemmas for the proof of Theorem 2. We begin with the following fundamental result of Sylvester and Erdős (see [3, Lemma 2] and [8, Lemma 1]).

Lemma 1. For $1 \leq i \leq t$, let $n+d_{i} d=B_{i} B_{i}^{\prime}$, where $B_{i}$ and $B_{i}^{\prime}$ are positive integers such that $P\left(B_{i}\right) \leq k$. Let $\mathcal{S}=\left\{B_{1}, \ldots, B_{t}\right\}$. For every prime $p \leq k$ with $\operatorname{gcd}(p, d)=1$, choose $B_{i} \in \mathcal{S}$ such that $p$ does not appear to a higher power in the factorisation of any other element of $\mathcal{S}$. Let $\mathcal{S}_{1}$ be the subset of $\mathcal{S}$ obtained by deleting from $\mathcal{S}$ all $B_{i_{p}}$ with $p \leq k$ and $\operatorname{gcd}(p, d)=1$. Then

$$
\prod_{B_{i} \in \mathcal{S}_{1}} B_{i} \leq(k-1) ! \prod_{p \mid d} p^{-\operatorname{ord}_{p}(k-1) !} .
$$

In the next lemma, we state estimates for $\pi(x)$ and a lower bound for $\operatorname{ord}_{p}(k-1)$ ! for applying $(7)$.

Lemma 2. (a) We have

$$
\begin{array}{ll}
\pi(x) \leq \frac{x}{\log x}+\frac{1.5 x}{\log ^{2} x} & \text { for } x>1, \\
\pi(x)>\frac{x}{\log x} & \text { for } x \geq 17 .
\end{array}
$$

(b) For a prime $p$,

$$
\operatorname{ord}_{p}(k-1) ! \geq \frac{k-p-1}{p-1}-\frac{\log (k-1)}{\log p} .
$$


Proof. The estimates on $\pi(x)$ in Lemma 2(a) are due to Rosser and Schoenfeld [7, p. 69]. For Lemma 2(b), we have

$$
\operatorname{ord}_{p}(k-1) !=\left[\frac{k-1}{p}\right]+\ldots+\left[\frac{k-1}{p^{r}}\right]
$$

where $p^{r} \leq k-1<p^{r+1}$. Thus

$$
\operatorname{ord}_{p}(k-1) ! \geq \frac{k-1}{p-1}\left(1-\frac{1}{p^{r}}\right)-r \geq \frac{k-p-1}{p-1}-\frac{\log (k-1)}{\log p} .
$$

The next result is a key lemma for computations in the proof of Theorem 2 .

Lemma 3. Let the assumptions of Theorem 2 be satisfied. Let $p_{1}, \ldots, p_{r_{1}}$ be a non-empty set of primes dividing $\Delta$. For $0 \leq i<k$ define

$$
G_{1}(i)=\omega(n+i d)-r_{2}(i)
$$

where $r_{2}(i)$ is the number of primes from $\left\{p_{1}, \ldots, p_{r_{1}}\right\}$ dividing $n+i d$. Let

$$
G_{2}=\max _{0 \leq i<k}\left(G_{1}(i)\right)
$$

and $r_{3}$ be the number of $i$ 's with $0 \leq i<k$ such that $G_{2}=G_{1}(i)$. Let $R=\left[\frac{1}{5} \pi(k)\right]+\pi(k)-\pi_{d}(k)+1$ and

$$
G_{3}=\omega\left(\Delta_{0}\right)-R G_{2}, \quad G_{3}^{\prime}=\omega\left(\Delta_{0}\right)-R G_{2}+R-r_{3} .
$$

Then

$$
\begin{aligned}
& G_{3} \leq \pi_{d}(k), \\
& G_{3}^{\prime} \leq \pi_{d}(k) \quad \text { if } r_{3} \leq R .
\end{aligned}
$$

Proof. Let $\Delta$ be obtained from $\Delta_{0}$ by deleting $r \leq R$ terms $n+i_{1} d$, $\ldots, n+i_{r} d$, say. For $1 \leq i \leq r_{1}$, set $\alpha_{i}=\operatorname{ord}_{p_{i}}\left(\Delta_{0}\right)$ and $\alpha_{i_{h}}=\operatorname{ord}_{p_{i}}\left(n+i_{h} d\right)$ with $1 \leq h \leq r$. Further let $\Delta_{0}=\gamma \gamma^{\prime}$ and $n+i_{h} d=\gamma_{h} \gamma_{h}^{\prime}$ for $1 \leq$ $h \leq r$ where $\gamma=p_{1}^{\alpha_{1}} \ldots p_{r_{1}}^{\alpha_{r_{1}}}, \gamma_{h}=p_{1}^{\alpha_{1 h}} \ldots p_{r_{1}}^{\alpha_{r_{1} h}}$ and $\operatorname{gcd}\left(\gamma^{\prime}, p_{1} \ldots p_{r_{1}}\right)$ $=\operatorname{gcd}\left(\gamma_{h}^{\prime}, p_{1} \ldots p_{r_{1}}\right)=1$. We have

$$
\Delta=\frac{\Delta_{0}}{\prod_{h=1}^{r}\left(n+i_{h} d\right)}=p_{1}^{\alpha_{1}-\sum_{h=1}^{r} \alpha_{1 h}} \ldots p_{r_{1}}^{\alpha_{r_{1}}-\sum_{h=1}^{r} \alpha_{r_{1} h}} \frac{\gamma^{\prime}}{\prod_{h=1}^{r} \gamma_{h}^{\prime}} .
$$

We observe that $\alpha_{i}>\sum_{h=1}^{r} \alpha_{i h}$ for $1 \leq i \leq r_{1}$, since each prime $p_{i}$ divides $\Delta$. Thus

$$
\omega(\Delta) \geq r_{1}+\omega\left(\gamma^{\prime}\right)-\sum_{h=1}^{r} \omega\left(\gamma_{h}^{\prime}\right) .
$$

We note that $r_{1}+\omega\left(\gamma^{\prime}\right)=\omega\left(\Delta_{0}\right)$ and $\omega\left(\gamma_{h}^{\prime}\right)=G_{1}\left(i_{h}\right)$. Hence from (10) we 
get

$$
\omega(\Delta) \geq \omega\left(\Delta_{0}\right)-\sum_{h=1}^{r} G_{1}\left(i_{h}\right)
$$

Hence

$$
\omega(\Delta) \geq \omega\left(\Delta_{0}\right)-r G_{2} \geq \omega\left(\Delta_{0}\right)-R G_{2}=G_{3} .
$$

Now the first assertion follows by (5). Let now $r_{3} \leq R$. Then we see that

$$
\sum_{h=1}^{r} G_{1}\left(i_{h}\right) \leq\left(R-r_{3}\right)\left(G_{2}-1\right)+r_{3} G_{2},
$$

which, together with (11), gives the result by (5).

3. Proof of Theorem 2. We observe from (5) that $\pi_{d}(k)>0$. Let $R$ be as in Lemma 3 so that $t \geq k-R$. For every prime $p$ dividing $\Delta$, we delete a term $n+d_{i} d$ of $\Delta$ in which $p$ appears to the maximum power. Then, by (5), we are left with a set $T$ of $n+d_{i} d$ having at least $t-\pi_{d}(k)$ terms of $\Delta$. Hence by Lemma 1,

$$
\prod_{i=0}^{t-\pi_{d}(k)-1}(n+i d) \leq \prod_{n+d_{i} d \in T}\left(n+d_{i} d\right) \leq(k-1) ! \prod_{p \mid d} p^{-\operatorname{ord}_{p}(k-1) !} .
$$

On the other hand,

$$
\begin{aligned}
& \prod_{i=0}^{t-\pi_{d}(k)-1}(n+i d) \\
& \quad \geq\left\{\begin{array}{l}
\left(t-\pi_{d}(k)-1\right) ! d^{t-\pi_{d}(k)-1} \\
\alpha(\alpha+1) \ldots\left(\alpha+t-\pi_{d}(k)-1\right) d^{t-\pi_{d}(k)} \quad \text { if } n \geq \alpha d \text { with } \alpha>0 .
\end{array}\right.
\end{aligned}
$$

Therefore

$$
d^{t-\pi_{d}(k)-1} \leq(k-1) \ldots\left(t-\pi_{d}(k)\right) \prod_{p \mid d} p^{-\operatorname{ord}_{p}(k-1) !}
$$

and for any integer $\alpha>0$ with $\alpha \leq k-t-1+\pi_{d}(k)$, we have

$$
\begin{aligned}
d^{t-\pi_{d}(k)} \leq & (\alpha-1) !(k-1) \ldots\left(\alpha+t-\pi_{d}(k)\right) \\
& \times \prod_{p \mid d} p^{-\operatorname{ord}_{p}(k-1) !} \quad \text { if } n \geq \alpha d .
\end{aligned}
$$

Thus by (12) we have,

$$
d^{t-\pi_{d}(k)-1} \leq(k-1) \ldots\left(t-\pi_{d}(k)\right)
$$

and

$$
d \leq(k-1)^{(1.2 \pi(k)+1) /(k-1.2 \pi(k)-2)} \prod_{p \mid d} p^{-\operatorname{ord}_{p}(k-1) ! /(k-1.2 \pi(k)-2)}
$$


By Lemma 2 and (15), it follows that for $k \geq 17$,

$$
\begin{aligned}
d \leq & \exp \left[\frac{1.2\left(1+\frac{1.5}{\log k}\right)+\frac{\log k}{k}}{1-1.2\left(\frac{1}{\log k}+\frac{1.5}{\log ^{2} k}\right)-\frac{2}{k}}\right] \\
& \times \prod_{p \mid d} p^{-\left(\frac{k-p-1}{p-1}-\frac{\log (k-1)}{\log p}\right) /\left(k-1.2 \frac{k}{\log k}-2\right)}
\end{aligned}
$$

which implies

$$
d \leq \exp \left[\frac{1.2\left(1+\frac{1.5}{\log k}\right)+\frac{\log k}{k}}{1-1.2\left(\frac{1}{\log k}+\frac{1.5}{\log ^{2} k}\right)-\frac{2}{k}}\right] .
$$

We use the inequalities (12)-(17) at several places. While using the inequalities (12)-(14), for a given $(d, k)$ we take the minimal value of $t$, that is, $t=k-R$. We observe that if any inequality (12)-(14) is not valid for $t=k-R$, then it is not valid for any $t>k-R$.

First we restrict to $k \geq 14$. If $k \geq 42$ then by (17), we find that $d \leq 34$. We use (14) to check that $d \leq 34$ for $14 \leq k<42$. Thus $d \leq 34$ for $k \geq 14$. For a given $d$ with $3 \leq d \leq 34$, we proceed as follows. We first use (16) to get a bound for $k$, say $k_{0}(d)$. Then for $14 \leq k \leq k_{0}(d)$, we apply (12) with actual values of $\pi_{d}(k)$ to find that $k \in U_{d}$ where $U_{d}$ is the set of integers $k \geq 14$ which are given in Table 2 whenever it is not empty. Let $k \in U_{d}$. We use (13) for this $k$ with suitable choice of $\alpha$ satisfying $0<\alpha \leq k-t-1+\pi_{d}(k)$ to get $n \leq \alpha d-1=: n_{d}(\alpha)$. We list in Table 2 those values of $d$ for which $U_{d}$ is not empty, $k_{0}(d)$, the set $U_{d}, \alpha$ and $n_{d}(\alpha)$.

Note that each $d$ in Table 2 is a prime or prime power whence $\pi(k)-$ $\pi_{d}(k) \leq 1$. We check that $2 \mid \Delta$ if $\operatorname{gcd}(d, 2)=1, k \geq 14 ; 3 \mid \Delta$ if $\operatorname{gcd}(d, 3)=1$, $k \geq 14$ and $5 \mid \Delta$ if $\operatorname{gcd}(d, 5)=1, k \geq 20$. Thus for the values of $d$ in Table 2 we take in Lemma 3 ,

$$
\begin{array}{ll}
r_{1}=3, & \left(p_{1}, p_{2}, p_{3}\right)=(2,3,5) \text { if } k \geq 20 \text { and } d \in\{7,11,13\}, \\
r_{1}=2, & \left(p_{1}, p_{2}\right)=(2,5) \text { if } k \geq 20 \text { and } d=3, \\
r_{1}=2, & \left(p_{1}, p_{2}\right)=(3,5) \text { if } k \geq 20 \text { and } d=4, \\
r_{1}=2, & \left(p_{1}, p_{2}\right)=(2,3) \text { if } k \geq 14 \text { and } d=5 \text { and if } \\
& 14 \leq k \leq 19 \text { and } d \in\{7,11,13,17,19,23,29,31\}, \\
r_{1}=1, & p_{1}=2 \text { if } 14 \leq k \leq 19 \text { and } d=3,9, \\
r_{1}=1, & p_{1}=3 \text { if } 14 \leq k \leq 19 \text { and } d=4,8 .
\end{array}
$$

For any given tuple $(n, d, k)$, we first compute $G_{3}$ and check (8). For example, let $(n, d, k)=(1,31,14)$. We find that $\omega\left(\Delta_{0}\right)=14, G_{2}=2, R=2$ and $\pi_{d}(k)=6$. Then $G_{3}=10>\pi_{d}(k)$. Thus (8) does not hold and the tuple $(1,31,14)$ is excluded. Likewise, we exclude all the tuples given by Table 2 except about 50 tuples satisfying (8). Now we compute $r_{3}$ for each of these 
Table 2

\begin{tabular}{|c|c|c|c|c|}
\hline$d$ & $k_{0}(d)$ & $U_{d}$ & $\alpha$ & $n_{d}(\alpha)$ \\
\hline 3 & 2900 & $\begin{array}{c}14-28 \\
29-341,349-370,373-375 \\
379-405,409-414,419-424, \\
433,434,439-459,461-489, \\
491-495,499-514,523-525 \\
619,659-666,691-693\end{array}$ & $\begin{array}{l}4 \\
8\end{array}$ & $\begin{array}{l}11 \\
23\end{array}$ \\
\hline 4 & 200 & $14-24,29-32,47,48$ & 2 & 7 \\
\hline 5 & 330 & $\begin{array}{c}14-26,29-55,59,61,62 \\
71-75,79,80,83,113-116\end{array}$ & 4 & 19 \\
\hline 7 & 170 & $14-25,29-34,47-49$ & 3 & 20 \\
\hline 8 & 70 & 14 & 1 & 7 \\
\hline 9 & 70 & 14,17 & 1 & 8 \\
\hline 11 & 100 & $14-21,31$ & 1 & 10 \\
\hline 13 & 90 & $14,15,17,19,20,23$ & 1 & 12 \\
\hline 17 & 70 & $14,15,17,19$ & 1 & 16 \\
\hline 19 & 70 & $14,15,17,19$ & 1 & 18 \\
\hline 23 & 55 & $14,17,19$ & 1 & 22 \\
\hline 29 & 50 & 14 & 1 & 28 \\
\hline 31 & 46 & 14 & 1 & 30 \\
\hline
\end{tabular}

remaining tuples. If $r_{3} \leq R$, we compute $G_{3}^{\prime}$ and check (9). For example, let $(n, d, k)=(1,4,17)$. We find $\omega\left(\Delta_{0}\right)=12, G_{2}=2, R=3, \pi_{d}(k)=6$ and $r_{3}=1<R$. Hence $G_{3}^{\prime}=8>\pi_{d}(k)$. Thus (9) does not hold and the tuple $(n, d, k)=(1,4,17)$ is excluded. Likewise all the remaining tuples are excluded except $(n, d, k)=(1,3,19)$. In this case $2 \mid \Delta, \omega\left(\Delta_{0}\right)=11, G_{2}=2$, $R=3, r_{3}=1, \pi_{d}(k)=7$ and hence $G_{3}=5<G_{3}^{\prime}=7=\pi_{d}(k)$. Thus both (8) and (9) hold. But we observe that 5 divides 4 terms in $\Delta_{0}$ and $R<4$. Hence $5 \mid \Delta$. Thus in Lemma 3 , we take $r_{1}=2,\left(p_{1}, p_{2}\right)=(2,5)$. We find $G_{2}=1 . G_{3}=11-3=8>\pi_{d}(k)$, a contradiction to (8). Thus all tuples in Table 2 are excluded.

Now we consider $d=2$. By (5), we have

$$
\pi(n+2 k-2)-\pi(n-1) \leq R+\pi_{d}(k) \leq \frac{6}{5} \pi(k)+1 .
$$

Hence

$$
\pi(2 k-1) \leq \pi(n-1)+\frac{6}{5} \pi(k)+1
$$


Suppose $n<2\left(\pi(k)+\left[\frac{1}{5} \pi(k)\right]\right)$. We use Lemma 2(a) in (19) to find $k \leq 6056$. Then we check that (19) is not valid for $322 \leq k \leq 6056$ using exact values of $\pi(x)$. Thus we may assume that $k \leq 321$. For these values of $n$ and $k$, we first observe that

$$
15 \mid \Delta \text { if } k \geq 20 \text { and } 3 \mid \Delta \text { if } 14 \leq k \leq 19 .
$$

Hence we take $r_{1}=2,\left(p_{1}, p_{2}\right)=(3,5)$ if $k \geq 20$ and $r_{1}=1, p_{1}=3$ if $14 \leq k \leq 19$. All the tuples other than

$$
(n, d, k) \in\{(1,2,14),(3,2,17),(1,2,18),(1,2,19),(3,2,19)\}
$$

are excluded as above by checking (8) and (9). Now we consider the tuples mentioned above other than $(1,2,14)$. We find that $5 \mid \Delta$ in all these cases and we use this additional property to check that (9) does not hold. Hence the only tuple which remains is $(n, 2, k)=(1,2,14)$ and we check that $\delta \in\{11,13,17,19,23\}_{3}$.

Thus we may assume that $n \geq 2\left(\pi(k)+\left[\frac{1}{5} \pi(k)\right]\right) \geq 2\left(k-t-1+\pi_{d}(k)\right)$. Now we use (13) with $\alpha=k-t-1+\pi_{d}(k)$. We get

$$
2^{k-\pi(k)-\left[\frac{1}{5} \pi(k)\right]-1} \leq\left(\pi(k)+\left[\frac{1}{5} \pi(k)\right]-1\right) !(k-1) 2^{-\operatorname{ord}_{2}(k-1) !} .
$$

Now we use Lemma 2(a) to derive

$$
\begin{aligned}
2 \leq & \exp \left[\frac{\left(1.2+\frac{1.8}{\log k}\right)\left(1+\frac{1}{\log k} \log \left(\frac{1.2}{\log k}+\frac{1.8}{\log ^{2} k}\right)\right)+\frac{\log k}{k}}{1-\frac{1.2}{\log k}-\frac{1.8}{\log ^{2} k}-\frac{1}{k}}\right] \\
& \times 2^{-\left(k-3-\frac{\log (k-1)}{\log 2}\right) /\left(k-1.2 \frac{k}{\log k}\right)}
\end{aligned}
$$

for concluding $k \leq 491$. Then we use (20) to find that $k<14$. This is a contradiction. This completes the proof of Theorem 2 for $k \geq 14$.

Finally, we consider $6 \leq k \leq 13$. Let $k=6$. Then $t \geq 2+\pi_{d}(k)$. Now we apply an argument of Lemma 1 . By (5), the product of the $t$ terms $n+d_{i} d$ of $\Delta$ are composed of at most $\pi_{d}(k)$ primes. Corresponding to each prime, we remove a term of $\Delta$ in which the prime appears to the maximum power. Then we are left with at least 2 terms say, $n_{1}$ and $n_{2}$. If a prime $p$ divides $n_{1}$ or $n_{2}$, then $p \in\{2,3,5\}$. Further we observe that either $5 \nmid n_{1}$ or $5 \nmid n_{2}$. Thus we get $n \leq 2^{2} \cdot 3=12$ and $n+d \leq 2^{2} \cdot 3 \cdot 5 \leq 60$. By similar argument, we see that $n \leq 12, n+d \leq 60$ for $k=7$. Now we use (12) to exclude several values of $d$. Thus we obtain $d \in V_{k}$ (see Table 3 ). Let now $8 \leq k \leq 13$. For each $k$, we use (14) to bound $d$. Then we use (12) as before to exclude several values of $d$. Finally, we use (13) for a suitable $\alpha$ to show that $n<\alpha d=: n_{k, d}$.

We list, in Table 3 below, the set $V_{k}$ and the bound $n<n_{k, d}$ for each value of $k$ with $6 \leq k \leq 13$. 


\section{Table 3}

\begin{tabular}{|c|c|c|}
\hline$k$ & $V_{k}$ & $n_{k, d}$ \\
\hline \multirow[t]{5}{*}{13} & 2,3 & $5 d$ \\
\hline & 5,7 & $4 d$ \\
\hline & 13 & $3 d$ \\
\hline & $4,11,17,19$ & $2 d$ \\
\hline & $8,9,25$, primes in $[23,61]$ & $d$ \\
\hline \multirow[t]{4}{*}{12} & 2 & $4 d$ \\
\hline & 3 & $3 d$ \\
\hline & $4,5,7$ & $2 d$ \\
\hline & 8,9, primes in $[11,31]$ & $d$ \\
\hline \multirow[t]{5}{*}{11} & 2 & $5 d$ \\
\hline & 3 & $4 d$ \\
\hline & $5,7,11,13$ & $3 d$ \\
\hline & $4,17,19$ & $2 d$ \\
\hline & $8,9,25$, primes in $[23,83]$ & $d$ \\
\hline 10 & $2,3,5,11$ & $d$ \\
\hline \multirow[t]{2}{*}{9} & 3 & $2 d$ \\
\hline & $2,5,7,9,11,13,17$ & $d$ \\
\hline \multirow[t]{3}{*}{8} & 2 & $3 d$ \\
\hline & 3,5 & $2 d$ \\
\hline & $4,7,8,9$, primes in $[11,47]$ & $d$ \\
\hline 7 & $\begin{array}{c}d \in[2,59] \\
d \notin\{6,10,12,18,20,24,30,36 \\
40,42,45,46,48,50,52,54,56,58\}\end{array}$ & 13 \\
\hline 6 & $\begin{array}{c}\text { Primes in }[2,59], 4,8,9,14, \\
21,27,33,39,49\end{array}$ & 13 \\
\hline
\end{tabular}

For the values of $(n, d, k)$ with $d \in V_{k}, n<n_{k, d}$ mentioned in Table 3, we check that

$$
\begin{aligned}
& 6 \mid \Delta \quad \text { if } k=6, d \text { prime } \geq 7 \text { and } d=49, \\
& \text { if } k=7, d \text { prime } \geq 11, \\
& \text { if } k=8, d \text { prime } \geq 11, \\
& \text { if } k=9, d=11,13,17, \\
& \text { if } k=10, d=5,11, \\
& \text { if } k=11, d \text { prime } \geq 13, \\
& \text { if } k=12, d \text { prime } \geq 5, \\
& \text { if } k=13, d \text { prime } \geq 5 \text { and } d=25 .
\end{aligned}
$$


Further, we get

$$
\begin{aligned}
2 \mid \Delta \quad & \text { if } k=6, d \in\{3,5,9,21,27,33,39\}, \\
& \text { if } k=7, d \in\{3,5,7,9,25,27,33,39,49,51,55,57\}, \\
& \text { if } k=8, d \in\{3,5,7,9\}, \\
& \text { if } k=9, d \in\{3,5,7,9\}, \\
& \text { if } k=10, d=3, \\
& \text { if } k=11, d \in\{3,5,7,9,11,25\}, \\
& \text { if } k=12, d \in\{3,9\}, \\
& \text { if } k=13, d \in\{3,9\},
\end{aligned}
$$

and

$$
\begin{aligned}
3 \mid \Delta \quad & \text { if } k=9, d=2, \\
& \text { if } k=10, d=2, \\
& \text { if } k=12, d=2,4,8, \\
& \text { if } k=13, d=2,4,8 .
\end{aligned}
$$

We do not have any such divisibility property for $k=6, d \in\{2,4,8,14\}$; $k=7, d \in\{2,4,8,14,15,16,21,22,26,28,32,34,35,38,44\} ; k=8, d \in$ $\{2,4,8\} ; k=11, d \in\{2,4,8\}$. For any given $(n, d, k)$ from Table 3 , we proceed as earlier. There are tuples for which both (8) and (9) hold. For these tuples we derive from (5) that $2 \mid \Delta$ if $2 \nmid d, 3 \mid \Delta$ if $3 \nmid d$ and $5 \mid \Delta$ if $5 \nmid d$. Using this we check now that either (8) or (9) does not hold. We exclude all the tuples but 13 of them. These are listed in Table 1.

4. Proofs of Theorem 1 and Corollaries $\mathbf{1}, \mathbf{2}$. We begin with the proof of Theorem 1. Assume that $k \geq 6$ and (4) does not hold. We observe that the number of primes $\leq k$ and dividing $\Delta_{0}$ is exactly $\pi_{d}(k)$. Hence there are at most $R=\pi(k)-\pi_{d}(k)+\left[\frac{1}{5} \pi(k)\right]+1$ primes $>k$ dividing $\Delta_{0}$. Each of these primes divides at most one term of $\Delta_{0}$. Deleting these terms we find distinct integers $d_{1}, \ldots, d_{t}$ in $[0, k)$ such that $t \geq k-R$ and

$$
\omega\left(\Delta\left(n, d, k, d_{1}, \ldots, d_{t}\right)\right) \leq \pi_{d}(k) .
$$

Hence by Theorem 2 , we conclude that $(n, d, k)$ equals one of the tuples listed in Theorem 1. This completes the proof of Theorem 1.

Suppose that the assumptions of Corollary 2 are satisfied. Then there are at most $\pi(k)-\pi_{d}(k)$ primes $>k$ dividing $\Delta$. We obtain a product $\Delta^{\prime}$ by omitting a term in $\Delta$ for each of these primes as in the proof of Theorem 1 such that $\omega\left(\Delta^{\prime}\right) \leq \pi_{d}(k)$. Now we apply Theorem 2 to $\Delta^{\prime}$. We conclude that the tuples $(n, d, k)$ take values as in Table 1. For each of these tuples $(n, d, k)$, we determine all the possible choices of $\delta$ such that $\omega(\Delta) \leq \pi(k)$ and $t \geq k-\left[\frac{1}{5} \pi(k)\right]-1$. We find that $(n, d, k, \delta) \in V_{1}$. 
Now we turn to the proof of Corollary 1. Let $4 \leq k<11$. Then the assertion follows by the result of Saradha and Shorey [8] mentioned in the introduction. For $k \geq 11$, we derive from Theorem 1 that either there are at least $\left[\frac{1}{5} \pi(k)\right]+2$ primes $\geq k$ dividing $\Delta_{0}$ or $(n, d, k)$ is one of the tuples in $\{(1,2,11),(1,3,11),(1,2,13),(3,2,13),(1,2,14)\}$ which are excluded by direct checking. Thus it remains to consider the case $k=3$. Now we take $k=3$. Then we may assume that $\Delta_{0}=2^{a} p^{b}$ where $p \in\{3,5,7\}$. Suppose $n$ is odd; then $n+2 d$ is odd. Hence $n=1, n+2 d=p^{b}$ and $n+d=2^{a}$, which implies $2^{a+1}-p^{b}=1$. Thus $p=3, a=b=1$ and $p=7, a=2$, $b=1$. The first possibility implies $d=1$, which is a contradiction, and the second possibility gives $(n, d, k)=(1,3,3)$, which is excluded. Let $n$ be even. Then $n=2^{\alpha_{1}}, n+d=p^{b}, n+2 d=2^{a-\alpha_{1}}$ with $a>2 \alpha_{1}>0$. Hence $p^{b}=2^{\alpha_{1}-1}+2^{a-\alpha_{1}-1}$, implying $\alpha_{1}=1,(p, b, a)=(3,1,3),(3,2,5),(5,1,4)$. If $(p, b, a)=(3,1,3)$, then $d=1$, which is not possible. Thus $(n, d, k) \in$ $\{(2,7,3),(2,3,3)\}$, which are excluded.

5. Proof of Theorem 3. The proof depends on the following two results. The first one is the well known Prime Number Theorem for arithmetic progressions (see Estermann [4, Chapter 2]). The second one is an explicit version of [1, Lemma 2]. For $x \geq 3$ and positive integers $D$ and $M$ with $\operatorname{gcd}(D, M)=1$, let $\pi(x ; D, M)$ denote the number of primes $p$ not exceeding $x$ such that $p \equiv M(\bmod D)$. For any positive integer $n$, let $\phi(n)$ denote Euler's totient function.

Lemma 4. Let $A>0$ and $B>0$. For $x \geq 3, D \leq(\log x)^{A}$ and $h \geq$ $x(\log x)^{-B}$, we have

$$
\pi(x+h ; D, M)-\pi(x ; D, M) \leq \frac{h}{\log (x) \phi(D)}\left(1+C_{3}\left(\frac{\log \log x}{\log x}\right)\right)
$$

where $C_{3}$ is an effectively computable positive number depending only on $A$ and $B$.

Lemma 5. For $x \geq 2$ and positive integer $D$ with $2<D<x$, we have

$$
\sum_{\substack{m \leq x \\ \cos (m, D)=1}} \frac{1}{m} \leq \frac{\phi(D)}{D}(\log x+5.2 \log \log D+5.02) .
$$

Proof. For odd $D$, it is clear that $\omega(D) \leq \log D / \log 3$ and this implies that

$$
\omega(D) \leq 1.12 \log D \quad \text { for all } D .
$$

It is well known by a classical convexity argument that

$$
\left|\sum_{m \leq x} \frac{1}{m}-\log x-\gamma\right| \leq \frac{2}{x}
$$


with $\gamma=.5772156649 \ldots$ (Euler's constant), and we proceed as in [1, Lemma 2] to obtain

$$
\sum_{\substack{m \leq x \\ \operatorname{cd}(m, D)=1}} \frac{1}{m} \leq(\log x+\gamma) \frac{\phi(D)}{D}+\left|\sum_{d \mid D} \frac{\mu(d) \log d}{d}\right|+2 .
$$

Further

$$
\sum_{d \mid D} \frac{\mu(d) \log d}{d}=-\frac{\phi(D)}{D} \sum_{p \mid D} \frac{\log p}{p-1}
$$

and

$$
\frac{D}{\phi(D)}<e^{\gamma} \log \log D+2
$$

(see [6, Lemma 7 and Remark 2]) and

$$
p_{i}>i \log i
$$

(see [7, p. 69]). Also we derive from the second inequality in Lemma 2(a) that $p_{i} \leq i^{3 / 2}$ for $i \geq 3$. We understand that an empty sum is equal to zero. Thus, since $p-1 \geq \frac{12}{13} p$ for $p \geq 13$, we have

$$
\begin{aligned}
\sum_{p \mid D} \frac{\log p}{p-1} & \leq \sum_{i=1}^{\omega(D)} \frac{\log p_{i}}{p_{i}-1} \leq 2.21+\sum_{i=6}^{\omega(D)} \frac{\log p_{i}}{p_{i}-1} \\
& \leq 2.21+\frac{39}{24} \sum_{i=6}^{\omega(D)} \frac{1}{i} \\
& \leq 2.21+\frac{39}{24}\left(\sum_{i=1}^{\omega(D)} \frac{1}{i}-\left(1+\frac{1}{2}+\frac{1}{3}+\frac{1}{4}+\frac{1}{5}\right)\right) \\
& \leq \frac{39}{24}(\log \omega(D)+\gamma+.5)-1.5 \\
& \leq \frac{39}{24} \log \log D+.44 .
\end{aligned}
$$

By combining (21)-(25) we obtain the assertion of the lemma.

Now we turn to the proof of Theorem 3. We proceed as in [1, Proof of Theorem 2]. Let $n, d, k$ be positive integers satisfying (6). We may assume that $k \geq C_{1}$ with $C_{1}$ sufficiently large. Let $J=\left[k /(\log k)^{10}\right]$. We put $S_{1}=$ $\{n+J d, \ldots, n+(k-1) d\}$. Let $S_{2}$ denote the set of elements of $S_{1}$ whose greatest prime factor exceed $k$. We observe that every element of $S_{2}$ can be written as $p \lambda$ where prime $p>k, p \equiv n_{\lambda}(\bmod d)$ with $n_{\lambda}=n \bar{\lambda}, \lambda \bar{\lambda} \equiv 1$ 
$(\bmod d)$ and

$$
\lambda \leq \frac{n+(k-1) d}{p} \leq 2 d \leq 2 \log k
$$

by (6). Further we see that

$$
\left|S_{2}\right| \leq \sum_{\substack{\operatorname{gcd}(\lambda, d)=1 \\ \lambda \leq 2 d}}\left\{\pi\left(\frac{n+(k-1) d}{\lambda} ; d, n_{\lambda}\right)-\pi\left(\frac{n+J d}{\lambda} ; d, n_{\lambda}\right)\right\}
$$

Now we apply Lemma 4 with $A=2, B=1, D=d, M=n_{\lambda}, x=(n+J d) / \lambda$, $x+h=(n+(k-1) d) / \lambda$. We observe that

$$
\frac{n+J d}{\lambda} \geq \frac{k}{4(\log k)^{10}}
$$

since $J=\left[k /(\log k)^{10}\right]$, which together with $n / k \leq d$ implies that

$$
(k-1-J) d \log \left(\frac{n+J d}{\lambda}\right) \geq n+J d .
$$

Therefore, the assumptions of Lemma 4 are satisfied and we get

$$
\left|S_{2}\right| \leq \frac{k}{\log k} \cdot \frac{d}{\phi(d)}\left(1+C_{4} \frac{\log \log k}{\log k}\right) \sum_{\substack{\operatorname{gcd}(\lambda, d)=1 \\ \lambda \leq 2 d}} \frac{1}{\lambda}
$$

where $C_{4}$ is an effectively computable absolute constant. Now the assertion of Theorem 3 follows from Lemma 5.

\section{References}

[1] R. Balasubramanian and T. N. Shorey, Perfect powers in products of terms in an arithmetical progression IV, Contemp. Math. 210 (1998), 257-263.

[2] R. Balasubramanian, M. Langevin, T. N. Shorey and M. Waldschmidt, On the maximal length of two sequences of integers in arithmetic progressions with the same prime divisors, Monatsh. Math. 121 (1996), 295-307.

[3] P. Erdős and J. L. Selfridge, The product of consecutive integers is never a power, Illinois J. Math. 19 (1975), 292-301.

[4] T. Estermann, Introduction to Modern Prime Number Theory, Cambridge Tracts in Math. and Math. Physics 41, Cambridge Univ. Press, 1969.

[5] M. Langevin, Plus grand facteur premier d'entiers en progression arithmétique, Sém. Delange-Pisot-Poitou, 18e année, 1976/77, no. 3, 6 pp.

[6] P. Moree, On arithmetic progressions having only few different prime factors in comparison with their length, Acta Arith. 70 (1995), 295-312.

[7] B. Rosser and L. Schoenfeld, Approximate formulas for some functions of prime numbers, Illinois J. Math. 6 (1962), 64-94.

[8] N. Saradha and T. N. Shorey, Almost perfect powers in arithmetic progression, Acta Arith. 99 (2001), 363-388. 
[9] T. N. Shorey and R. Tijdeman, On the number of prime factors of a finite arithmetical progression, Sichuan Daxue Xuebao 26 (1989), 72-74.

[10] - - - On the greatest prime factor of an arithmetical progression, in: A Tribute to Paul Erdős, A. Baker, B. Bollobás and A. Hajnal (eds.), Cambridge Univ. Press, 1990, 385-389.

[11] J. J. Sylvester, On arithmetical series, Messenger Math. 21 (1892), 1-19, 87-120.

School of Mathematics

Tata Institute of Fundamental Research

Homi Bhabha Road

Mumbai 400 005, India

E-mail: saradha@math.tifr.res.in

shorey@math.tifr.res.in
Mathematical Institute

Leiden University

P.O. Box 9512

2300 RA Leiden, The Netherlands

E-mail: tijdeman@math.leidenuniv.nl

Received on 20.10.2000

and in revised form on 12.7.2001 